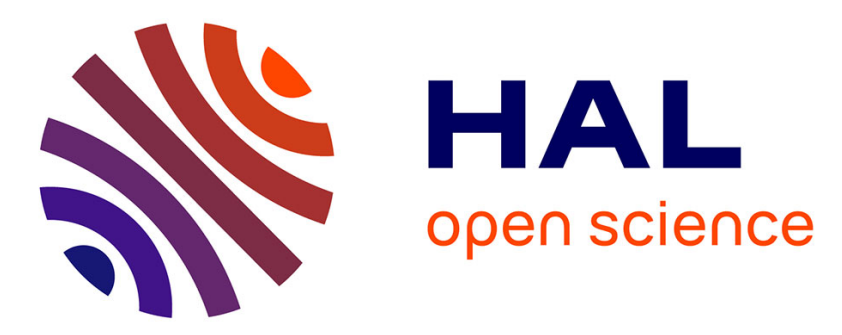

\title{
Détermination numérique des caractéristiques des générateurs impulsionnels à explosif gazeux. Application à un générateur de 10 mégajoules
}

R. Hahn, M. Legentil, C. Rioux

\section{To cite this version:}

R. Hahn, M. Legentil, C. Rioux. Détermination numérique des caractéristiques des générateurs impulsionnels à explosif gazeux. Application à un générateur de 10 mégajoules. Revue de Physique Appliquée, 1978, 13 (8), pp.405-414. 10.1051/rphysap:01978001308040500 . jpa-00244468

HAL Id: jpa-00244468

https://hal.science/jpa-00244468

Submitted on 1 Jan 1978

HAL is a multi-disciplinary open access archive for the deposit and dissemination of scientific research documents, whether they are published or not. The documents may come from teaching and research institutions in France or abroad, or from public or private research centers.
L'archive ouverte pluridisciplinaire HAL, est destinée au dépôt et à la diffusion de documents scientifiques de niveau recherche, publiés ou non, émanant des établissements d'enseignement et de recherche français ou étrangers, des laboratoires publics ou privés. 
Classification

Physics Abstracts

06.60

\title{
DÉTERMINATION NUMÉRIQUE DES CARACTÉRISTIQUES DES GÉNÉRATEURS IMPULSIONNELS A EXPLOSIF GAZEUX. APPLICATION A UN GÉNÉRATEUR DE 10 MÉGAJOULES
}

\author{
R. HAHN, M. LEGENTIL et C. RIOUX \\ Laboratoire de Physique des plasmas, Groupe électrotechnique et fusion contrôlée, \\ Université Paris-Sud, 91405 Orsay, France
}

(Reçu le 29 avril 1976, révisé le 20 avril 1978, accepté le 8 mai 1978)

\begin{abstract}
Résumé. - L'article a trait aux générateurs impulsionnels mettant en jeu l'expansion rapide d'un cylindre métallique parcouru par un courant axial intense. L'énergie est fournie chimiquement à l'aide d'un explosif gazeux. Il décrit les méthodes numériques permettant de déterminer leurs performances. Ces méthodes sont appliquées à la conception d'un générateur à basse impédance susceptible de délivrer une énergie de $10 \mathrm{MJ}$ en $100 \mu$ s sous la forme d'un courant de $30 \mathrm{MA}$. Le coût d'un tel générateur, de l'ordre de $10^{6} \mathrm{~F}$, serait incomparablement moins cher que les générateurs à explosifs solides ou les condensateurs. Son coût de fonctionnement serait de $10^{3} \mathrm{~F}$ par tir.
\end{abstract}

\begin{abstract}
This article describes pulsed generators in which a metallic cylinder flowed by an axial high current rapidly expands, driven by the explosion of a gaseous chemical explosive. Numerical methods determining their performance are given. These methods are applied for the design of a low-impedance generator capable of delivering an energy of $10 \mathrm{MJ}$ in $100 \mu \mathrm{s}$ with a current of $30 \mathrm{MA}$. The cost of such a generator $\left(\sim 10^{6} \mathrm{~F}\right)$ would be very much less than equivalent solid explosives generators or condensator banks. Its working cost would be $10^{3} \mathrm{~F}$ per operation.
\end{abstract}

1. Introduction. - La plupart des procédés permettant de produire des impulsions électromagnétiques courtes de grande énergie (par exemple de $10 \mathrm{MJ}$ au moins en quelques $\mu \mathrm{s}$ ) sont très onéreux soit par l'investissement (condensateurs), soit par le coût de fonctionnement (générateurs à explosifs solides). Pour obtenir des sources d'énergie de ce type à l'aide d'investissements modérés, nous nous sommes orientés vers des générateurs à explosifs employant des structures nouvelles, où un explosif doux constitué par un gaz détonnant est substitué aux explosifs brisants des générateurs à explosifs usuels [1].

Par cette méthode, le prix de revient de l'explosif (mélange $\mathrm{O}_{2}+2 \mathrm{H}_{2}$ produit par électrolyse directe de l'eau) est négligeable. D'autre part, les parties mécaniques détruites à chaque fonctionnement $d u$ générateur sont peu importantes et de remplacement aisé. Enfin, la production du mélange explosif sur le site même de l'emploi évite les difficiles problèmes de protection liés au stockage d'explosifs solides. L'emploi d'explosifs gazeux, qui permet les consi- dérables avantages pratiques énoncés ci-dessus, limite à $600 \mathrm{~m} / \mathrm{s}$ environ la vitesse des pièces métalliques comprimant le champ. Cette valeur, faible par rapport aux $2 \mathrm{~km} / \mathrm{s}$ des générateurs à explosifs solides, allonge a priori le temps de fourniture de l'énergie. Pour que les temps restent cependant du même ordre, nous avons choisi pour les dispositifs à explosifs gazeux une structure assez sensiblement différente de celle des systèmes à explosifs solides. Dans notre cas, l'onde de détonation, de forme cylindrique, accélère simultanément toutes les pièces métalliques en mouvement.

Les principes de fonctionnement de ces générateurs nous ayant entraînés vers des procédés technologiques nouveaux, nous nous sommes proposés dans un premier temps de tester ces concepts sur un dispositif à faible énergie [2]. Les résultats expérimentaux obtenus nous ont permis de vérifier que certaines inconnues liées au comportement des matériaux (résistance mécanique d'un métal en dilatation rapide, taux de dilatation avant rupture) ou certaines approximations 
de calculs (incompressibilité du métal implosé, faible échauffement joule du métal, etc...) laissaient à la détermination des performances une précision largement suffisante pour permettre la conception de dispositifs à plus hautes caractéristiques.

A la suite de ces enseignements, nous nous proposons dans le présent article d'exposer les possibilités potentielles de ce type de générateur à un niveau d'énergie plus élevé, à partir de laquelle ils semblent particulièrement intéressants, c'est-à-dire au-delà de 10 à $20 \mathrm{MJ}$.

Dans un premier temps, nous discutons les conditions de validité des hypothèses retenues, en montrant notamment que certaines indéterminations physiques (dilatation volumique du tube métallique explosé, résistances de contact, etc...) sont comprises dans des limites assez étroites pour ne pas modifier les conclusions tirées d'hypothèses simplificatrices convenablement choisies. L'étude littérale approchée des performances, exposée dans le précédent article, était destinée à guider la recherche des structures optimales à tous niveaux d'énergie; nous donnons ici la méthode d'approche numérique plus précise que nous avons employée pour obtenir le fonctionnement détaillé d'un générateur dont les dimensions sont données à l'avance. La conception et le fonctionnement d'un générateur de $10 \mathrm{MJ}$ d'énergie utile sont étudiés ensuite en conservant les dispositions techniques expérimentées sur le petit modèle, excepté en ce qui concerne le tube métallique explosé où une solution technologique plus prometteuse a priori est proposée et adoptée dans le calcul des caractéristiques. Enfin, comme le taux de multiplication de l'énergie de ces générateurs est faible (par exemple de l'ordre de 5), la masse et donc le prix des condensateurs donnant le courant initial risqueraient d'être prohibitifs. Pour diminuer leur importance, nous examinons les problèmes liés à la mise en cascade de plusieurs générateurs. Enfin, nous terminons par une étude économique globale indicative permettant de comparer le système à des procédés plus usuels.

2. Modèle et hypothèses de calcul. - Rappelons tout d'abord la structure globale et le fonctionnement du dispositif représenté sur la figure 1 et déjà décrit [2]. A l'état initial, le condensateur de $2000 \mu \mathrm{F}$ est chargé et le tube cylindrique en aluminium est rempli du mélange explosif. L'amorçage de la détonation s'effectue par le fil explosé. Il en résulte une expansion rapide du cylindre, lequel déclenche plusieurs phénomènes physiques successifs. Tout d'abord, le tube touche mécaniquement les contacteurs, ce qui décharge le condensateur : le tube est parcouru par un courant intense. Lorsque le courant atteint sa valeur maximale, le circuit électrique est court-circuité par un nouveau contact du tube avec la carcasse. A partir de cet instant, un flux magnétique azimutal important est piégé entre le cylindre et la carcasse. Sa compres-

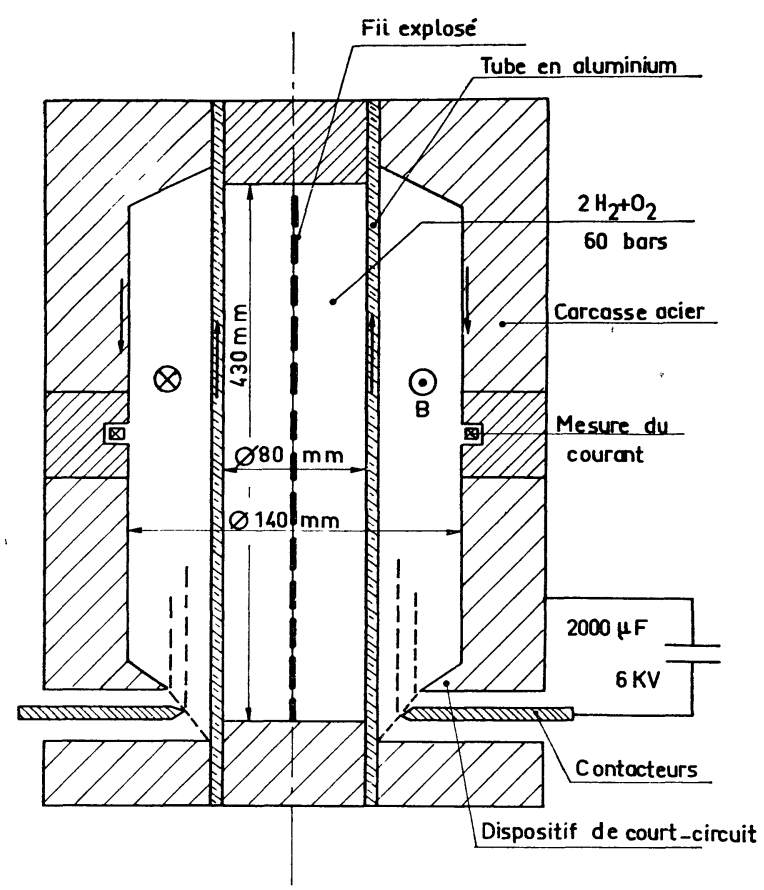

FIG. 1. - Schéma du générateur testé expérimentalement : le générateur de $10 \mathrm{MJ}$ aurait une structure analogue mais des dimensions supérieures.

[Scheme of the experimented generator : the $10 \mathrm{MJ}$ generator would have a similar structure but greater dimensions.]

sion freine le cylindre, ce qui transforme son énergie cinétique en énergie magnétique.

Pour effectuer l'étude, nous appellerons $\sigma_{1}, \rho_{1}$ la conductivité et la masse volumique du cylindre en aluminium (appelé liner) et $\sigma_{\mathrm{c}}, \rho_{\mathrm{c}}$ les valeurs correspondantes pour la carcasse. Les différents rayons $r$ mis en jeu au cours du fonctionnement sont représentés schématiquement sur la figure $2\left(r_{0}\right.$ et $r_{1}$, qui représentent les rayons intérieur et extérieur du liner, sont variables dans le temps).

La détermination numérique de l'évolution $\mathrm{du}$ système s'effectue à partir d'un certain nombre d'approximations. La première consiste à négliger la résistance électrique des raccords du court-circuit reliant le tube à la carcasse : ceci est justifié dès que la longueur des deux tubes est grande par rapport à

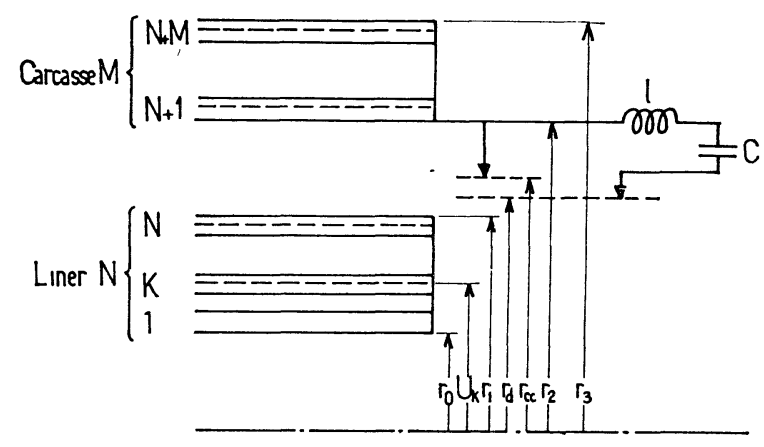

FIG. 2. - Modèle de calcul pour le générateur.

[Computation pattern.] 
leur intervalle. En effet, la résistance de contact cuivre-aluminium tombe à des valeurs très basses dès que la pression de contact entre les parties métalliques dépasse 2 ou $3 \mathrm{kgf} / \mathrm{mm}^{2}$ [12]. Dans le montage d'essai, la densité de courant initiale dans l'épaisseur de peau était de l'ordre de $200 \mathrm{kA} / \mathrm{cm}^{2}$. Alors,

$$
\frac{\text { résistance de contact }}{\text { résistance du circuit (en peau) }} \simeq 2 \% \text {. }
$$

Au cours de la compression du flux, l'accroissement de la densité de courant rend la résistance de contact plus négligeable encore. Pour un générateur de $10 \mathrm{MJ}$, la chute de tension au contact resterait inférieure à $1 \%$ dans tous les cas.

Au cours de l'implosion, le cylindre entre en déformation plastique rapide. Le comportement de l'aluminium dans de telles conditions est mal connu car il est soumis à un ensemble de contraintes mécaniques complexes : pression interne radiale à l'accélération de l'ordre du kilobar et pression externe au freinage de quelques kilobars, contraintes de traction azimutales élevées et assez inconnues au cours de la déformation plastique. Compte tenu du module d'élasticité de l'aluminium $\left(8 \times 10^{5}\right.$ bars $)$, les pressions radiales appliquées au tube n'engendrent que des compressions inférieures à $1 \%$. En revanche, la dilatation azimutale rapide produit un accroissement du volume du tube important mais difficile à évaluer. En remarquant qu'une modification même notable $(10 \%$ ?) des caractéristiques du tube dilaté (densité et résistivité notamment) n'engendrerait que de faibles modifications sur les caractéristiques globales du générateur, nous admettrons faute de données suffisamment précises que le volume du tube se conserve ainsi que sa résistivité.

Une inconnue physique importante est la contrainte de traction azimutale au cours de la dilatation rapide. Les essais en l'absence de champ sur le petit générateur [1] ont montré que cet effort atteint plus de 2 fois les valeurs obtenues en dilatation lente. Pour le générateur de $10 \mathrm{MJ}$, la vitesse de déformation plus faible réduit un peu cette contrainte. Il en est probablement de même dans les régions extérieures chauffées par le courant; nous avons néanmoins conservé la valeur expérimentale maximale pour calculer les pertes correspondantes.

Pour décrire le circuit électrique du générateur nous considérons que :

- Les deux tubes présentent toujours une parfaite symétrie de révolution, c'est-à-dire que dans un système d'axe cylindrique $(r, \theta, z)$, on a toujours

$$
\frac{\partial}{\partial \theta}=\frac{\partial}{\partial z}=0
$$

- Lorsque le rayon extérieur du liner $r(t)$ est compris entre $r_{\mathrm{d}}$ et $r_{\text {cc }}$, le liner, la carcasse, le banc de condensateurs de capacité $C$ chargé initialement à la tension $V_{0}$ et l'inductance $l$ (somme de l'inductance interne du banc et de ses liaisons) constituent une boucle fermée.

- Lorsque $r(t)$ dépasse la valeur $r_{\mathrm{cc}}$ le liner et la carcasse sont court-circuités sur eux-mêmes.

Le modèle utilisé pour le calcul est représenté sur la figure 2. Le liner est découpé en $N$ tranches coaxiales, la carcasse en $M$ tranches également coaxiales. Les tranches sont numérotées de 1 à $N$ pour le liner, et de $N+1$ à $N+M$ pour la carcasse. Nous pouvons choisir $N$ et $M$ assez grands pour considérer les couches comme infiniment minces et définies par leur rayon.

Nous allons exprimer, à partir des formes intégrales des équations de Maxwell, les relations entre les flux, les courants et leurs dérivées temporelles. La source d'énergie annexe créant le courant initial impose une tension $V$ aux bornes du générateur avant que celles-ci ne soient court-circuitées. Nous exprimerons cette tension $V$ à l'aide des flux, des courants, de la position et de la vitesse du liner. Cette vitesse est déterminée à partir des mesures de l'évolution temporelle du rayon extérieur du liner effectuées en absence de champ magnétique [2]. Le système d'équations différentielles ainsi obtenu sera résolu numériquement.

3. Les équations d'évolution. - Nous choisissons un découpage où les tranches successives ont la même masse, c'est-à-dire la même section :

$$
s_{1}=\frac{\pi}{N}\left(r_{1}^{2}-r_{0}^{2}\right)
$$

Chaque tranche, numérotée $K$, est définie par son rayon moyen $U_{K}$ (séparant la tranche elle-même en deux aires égales). En supposant le métal incompressible, il en résulte que

$U_{\mathbf{K}}^{2}=r_{0}^{2}+\frac{2 K-1}{2} \frac{s_{1}}{\pi}$ pour $K=1, \ldots, N$.

Pour la carcasse, on obtient les expressions correspondantes :

$$
\begin{gathered}
s_{\mathrm{c}}=\frac{\pi}{M}\left(r_{3}^{2}-r_{2}^{2}\right) \\
U_{\mathbf{K}}^{2}=r_{2}^{2}+\frac{2 K-2 N-1}{2} \cdot \frac{s_{\mathrm{c}}}{\pi}
\end{gathered}
$$

pour

$$
K=N+1, \ldots, N+M
$$

Exprimons la variation de flux $\Phi_{K}$ dans le circuit limité par deux génératrices correspondant aux rayons $U_{K}$ et $U_{N+M}$, mises en série soit directement si $K>N$, soit par l'intermédiaire d'une différence de potentiel extérieure $V$ si $K \leqslant N$. Rappelons que le rayon $U_{N+M}$ correspond à la dernière couche de la carcasse. 


$$
\begin{aligned}
& \dot{\Phi}_{K}=-\frac{J_{K}}{\sigma_{1}} \mathrm{~h}+\frac{J_{N+M}}{\sigma_{\mathrm{c}}} h+V \\
& \dot{\Phi}_{K}=-\frac{J_{K}}{\sigma_{\mathrm{c}}}+\frac{J_{N+M}}{\sigma_{\mathrm{c}}} h
\end{aligned}
$$

Les flux $\Phi_{K}$ sont calculés en supposant que le courant de la tranche $K$ est entièrement concentré dans une nappe cylindrique de rayon $U_{K}$ et vaut $s_{K} J_{K}$ (où $s_{K}$ prend les valeurs $s$ ou $s_{\mathrm{c}}$ suivant que la tranche considérée est située dans le liner $1 \leqslant K \leqslant N$ ou dans la carcasse $N+1 \leqslant K \leqslant N+M)$. L'induction $B$ varie donc en $r^{-1}$ entre deux nappes voisines. Le flux compris entre $U_{K}$ et $U_{K+1}$ s'écrit :

$$
\text { . } \Phi_{K}-\Phi_{K+1}=\frac{\mu_{0} h}{2 \pi}\left(\sum_{i=1}^{K} s_{i} J_{i}\right) \log \frac{U_{K+1}}{U_{K}}
$$

$$
\left.\begin{array}{l}
K=1, \ldots, N \\
K=N+1, \ldots, N+M-1
\end{array}\right\}
$$

$$
\begin{aligned}
J_{1} & =\frac{2 \pi}{\mu_{0} s_{1} h} \cdot \frac{\Phi_{1}-\Phi_{2}}{\log \frac{U_{2}}{U_{1}}} \quad \Phi_{0}=\Phi_{1} \\
J_{N+M} & =\frac{-2 \pi}{\mu_{0} s_{\mathrm{c}} h} \cdot \frac{\Phi_{N+M-1}}{\log \frac{U_{N+M}}{U_{N+M-1}}} \quad \Phi_{N+M+1}=\Phi_{N+M}=0 .
\end{aligned}
$$

CIRCUIT EXTÉRIEUR : EXPRESSION DE $V$. - Lorsque le circuit du générateur est alimenté par le banc de condensateurs dont la tension est $V_{c}$, la différence de potentiel $V$ à ses bornes est égale à :

$$
V=V_{\mathrm{c}}-l \frac{\mathrm{d} I}{\mathrm{~d} t} \text { pour } r_{\mathrm{d}} \leqslant r(t) \leqslant r_{\mathrm{cc}}
$$$$
\text { avec } \quad \frac{\mathrm{d} V_{\mathrm{c}}}{\mathrm{d} t}=-\frac{I}{c} \text {. }
$$

Lorsque le court-circuit avec la carcasse est effectué, on a :

$$
V=0 \quad \text { pour } \quad r_{\mathrm{cc}} \leqslant r(t) .
$$

Nous obtenons par différence avec l'expression analogue de $\Phi_{K-1}-\Phi_{K}$, pour $K=2, \ldots, N+M-1$ :

$$
J_{K}=\frac{2 \pi}{\mu_{0} s_{K} h}\left[\frac{\Phi_{K}-\Phi_{K+1}}{\log \frac{U_{K+1}}{U_{K}}}-\frac{\Phi_{K-1}-\Phi_{K}}{\log \frac{U_{K}}{U_{K-1}}}\right] .
$$

Aux tranches limites, nous avons :

Pour pouvoir intégrer les équations (5) où $V$ est donné par (10), il nous faut éliminer $\mathrm{d} I / \mathrm{d} t$.

Le courant total $I$ est la somme des courants $J_{K}$ donnés par la relation (7) :

$I=s_{1} \sum_{1}^{N} J_{K}=s_{\mathrm{c}} \sum_{N+1}^{N+M} J_{K}=\frac{2 \pi}{\mu_{0} h} \cdot \frac{\Phi_{N}-\Phi_{N+1}}{\log \frac{U_{N+1}}{U_{N}}}$.

Calculons $\mathrm{d} I / \mathrm{d} t$ à partir de cette équation (11) en exprimant $\dot{\Phi}_{N}$ et $\dot{\Phi}_{N+1}$ à l'aide des équations (5) et en tenant compte de $\mathrm{d} U_{N+1} / \mathrm{d} t=0$; nous obtenons :

Or d'après $(10 a)$

$$
\frac{\mathrm{d} I}{\mathrm{~d} t}=\frac{2 \pi}{\mu_{0} h \log \frac{U_{N+1}}{U_{N}}}\left(\frac{J_{N+1}}{\sigma_{\mathrm{c}}} h-\frac{J_{N}}{\sigma_{1}} h+U+\frac{\Phi_{N}-\Phi_{N+1}}{\log \frac{U_{N+1}}{U_{N}}} \cdot \frac{1}{U_{N}} \cdot \frac{\mathrm{d} U_{N}}{\mathrm{~d} t}\right) .
$$

$$
\frac{\mathrm{d} I}{\mathrm{~d} t}=\frac{V_{\mathrm{c}}-V}{l}
$$

Ces deux relations nous donnent $V$ :

$$
V=\frac{-l\left(\frac{J_{N+1}}{\sigma_{\mathrm{c}}} h-\frac{J_{N}}{\sigma_{1}} h+\frac{\Phi_{N}-\Phi_{N+1}}{\log \frac{U_{N+1}}{U_{N}}} \frac{1}{U_{N}} \frac{\mathrm{d} U_{N}}{\mathrm{~d} t}\right)+\frac{\mu_{0} h}{2 \pi} V \log \frac{U_{N+1}}{U_{N}}}{l+\frac{\mu_{0} h}{2 \pi} \log \frac{U_{N+1}}{U_{N}}} .
$$


Le calcul de la diffusion du champ magnétique dans le liner exige la connaissance à chaque instant de sa position et de sa vitesse (pour connaître les termes $U_{N}$ et $\mathrm{d} U_{N} / \mathrm{d} t$ ).

4. Calcul du mouvement du tube explosé. - L'énergie initiale totale (constituée par l'énergie interne de la vapeur d'eau provenant de la détonation du mélange $2 \mathrm{H}_{2}+\mathrm{O}_{2}$, et par l'énergie magnétique initiale) se transforme partiellement au cours du mouvement en énergie cinétique de déformation du liner, en énergie magnétique et en énergie joule, le reste étant toujours sous forme d'énergie interne de la vapeur d'eau.

Nous faisons pour évaluer ces énergies les approximations suivantes :

- le liner est un tube mince $r_{0} \simeq r_{1}$,

- la variation d'énergie interne de la vapeur d'eau $E-E_{0}$ ne dépend que du rapport de détente $\left(r(t) / r_{0}\right)^{2}$,

- l'énergie cinétique de la vapeur d'eau est négligeable,

- l'énergie de déformation du liner $W_{\mathrm{d}}$ ne dépend que de son allongement $r(t) / r_{1}$.

Avec ces approximations, la conservation de l'énergie s'exprime par :

$$
\begin{aligned}
& E\left(\frac{r(t)}{r_{0}}\right)-E_{0}+W_{\mathrm{d}}\left(\frac{r(t)}{r_{0}}\right)+\frac{1}{2} m v^{2}+ \\
& \quad+\frac{1}{2} L(t) \cdot I^{2}(t)-\frac{1}{2} L_{0} I_{0}^{2}+\int_{0}^{t} R I^{2} \mathrm{~d} t=0 .
\end{aligned}
$$

L'équation fondamentale de la dynamique s'en déduit en dérivant cette expression par rapport au rayon $r(t)$.

En l'absence de champ magnétique $(I=0)$, l'expression (15) permet de déterminer l'accélération $g$ du tube

$$
g=\frac{\mathrm{d}^{2} r}{\mathrm{~d} t^{2}}=-\frac{1}{m} \frac{\mathrm{d}}{\mathrm{d} r}\left(E\left(\frac{r(t)}{r_{0}}\right)+W_{\mathrm{d}}\left(\frac{r(t)}{r_{0}}\right)\right) .
$$

En présence de champ magnétique et en tenant compte de l'équation électrique du circuit

$$
\left(\frac{\mathrm{d}}{\mathrm{d} t}\left(\frac{1}{2} L I^{2}\right)+R I^{2}+\frac{1}{2} I^{2} \frac{\mathrm{d} L}{\mathrm{~d} t}=0\right),
$$

l'expression (15) entraîne :

$m \frac{\mathrm{d}^{2} r}{\mathrm{~d} t^{2}}=-\frac{\mathrm{d}}{\mathrm{d} r}\left(E\left(\frac{r(t)}{r_{0}}\right)+W_{\mathrm{d}}\left(\frac{r(t)}{r_{0}}\right)\right)+\frac{1}{2} \frac{I^{2}}{v} \frac{\mathrm{d} L}{\mathrm{~d} t}$.

Sachant que

$$
\frac{\mathrm{d} L}{\mathrm{~d} t}=-\frac{\mu_{0}}{2 \pi} h \cdot \frac{v}{r},
$$

l'équation du mouvement du liner s'écrit sous la forme :

$$
m \frac{\mathrm{d}^{2} r}{\mathrm{~d} t^{2}}=m \cdot g(r)-\frac{\mu_{0}}{4 \pi} \frac{h}{r} I^{2}(t) .
$$

Dans cette équation $g(r)$ est l'accélération du liner en l'absence de champ magnétique.

Remarque. - La dynamique résultant des hypothèses ci-dessus n'est pas très précise, surtout en raison du manque de données concernant la relation contrainte-déformation pour ce type de dilatation. Elle est cependant largement suffisante pour donner une bonne évaluation des performances globales.

5. Résolution numérique. - Le système d'équations différentielles à résoudre est donc constitué par les équations (5), (10), (7) et (14), les conditions aux limites étant :

$$
\varphi_{0}=\varphi_{1}, \quad \varphi_{N+M}=0 .
$$

L'accélération de la surface extérieure $g\left(U_{N}\right)$ du liner en absence de champ magnétique a été déterminée expérimentalement. Au cours de la compression du champ, $m$ étant la masse du liner, nous avons :

$$
\frac{\mathrm{d}^{2} U_{N}}{\mathrm{~d} t^{2}}=g\left(U_{N}\right)-\frac{\mu_{0} h}{4 \pi \cdot m} \frac{I^{2}(t)}{U_{N}} .
$$

Nous avons résolu numériquement ces équations. Remarquons que la forme des équations (5) et (7) est équivalente à un algorithme explicite pour l'équation de la diffusion. On sait que la stabilité de calcul des équations aux différences explicites correspondant à une équation de la diffusion exige [3] :

$$
\frac{2}{\mu_{0} \sigma} \cdot \frac{\Delta t}{(\Delta U)^{2}} \lesssim 1
$$

où $\Delta t$ et $\Delta U=\left(U_{\mathbf{K}+1}-U_{\mathbf{K}}\right)$ sont les maillages dans le temps et dans l'espace. Appliquons ce critère pour calculer le $\Delta U$ minimum lorsque le liner est en fin de course.

Avec un $\Delta t=10^{-7} \mathrm{~s}$ on obtient $\Delta U \gtrsim 7 \times 10^{-5} \mathrm{~m}$, ce qui pour un tube d'épaisseur $6 \mathrm{~mm}$ se dilatant 1,5 fois conduit à un nombre $N$ de tranches inférieur à 55 .

Nous avons alors intégré le système précédent en utilisant un programme d'intégration de la bibliothèque de l'UNIVAC d'Orsay : (Runge-Kutta d'ordre 5), avec $N=M=40, \Delta t=10^{-7} \mathrm{~s}$ assurant une précision de $10^{-6}$ sur un pas et environ $10^{-3}$ pour le calcul total, ce qui est largement suffisant compte tenu des approximations faites et des incertitudes dans les cotes de réalisation.

6. Résultats des calculs numériques. Comparaison à l'expérience. - Avec les paramètres et dimensions du prototype de générateur expérimenté [2], les résultats numériques et expérimentaux sont en très bon 
accord, ce qui confirme indirectement les hypothèses physiques que nous avons effectuées. Nous pouvons par suite extrapoler raisonnablement ce type de générateur à un niveau d'énergie plus élevé pour lequel les conditions physiques d'emploi des matériaux restent voisines. Nous avons à cet effet choisi le niveau $10 \mathrm{MJ}$, à notre sens caractéristique des possibilités potentielles de ces générateurs.

7. Conception d'un générateur de 10 MJ. - Avant de fixer les dimensions de ce générateur et d'étudier son fonctionnement, nous allons proposer des améliorations technologiques susceptibles d'être apportées au prototype expérimenté. Nous adopterons ensuite ces améliorations pour le calcul des performances.

7.1 AMÉLIORATIONS DU DISPOSITIF. - Pour obtenir un tube de faible masse permettant un faible coût d'exploitation, il est nécessaire de lui fournir une forte énergie spécifique. Or, par une suite simple de relations de proportionnalités, on peut montrer que l'énergie spécifique fournie au liner est de la forme :

$$
w=\frac{E-E_{0}}{m}=K_{\mathrm{L}} \cdot \frac{n}{\varpi} \cdot \frac{1}{T}
$$

(où $n$ est la contrainte mécanique dans le liner froid, $\varpi$ sa masse spécifique et $T$ la température absolue du mélange explosif ; le coefficient $K_{\mathrm{L}}$ est fonction de la nature du gaz et du rapport de détente $r_{\mathrm{f}} / r_{0}$ ).

Avec de l'aluminium A5 recuit, à la température ambiante, il n'est pas possible en pratique de dépasser une vitesse de l'ordre de $250 \mathrm{~m} / \mathrm{s}$ ( $30 \mathrm{~kJ} / \mathrm{kg}$ environ). Un abaissement de la température $T$ ne donnant qu'un accroissement assez faible de la vitesse (50 à $60 \%$ ). on peut s'orienter vers l'utilisation de matériaux admettant tout à la fois :

- une bonne résistance mécanique à froid,

- un fort allongement avant rupture,

- une bonne conductivité électrique pour limiter les fuites de flux.

Un tel matériau n'existant pas à notre connaissance. on propose de lui substituer un liner composite où seraient séparées les deux fonctions du tube : tenue mécanique de la pression initiale et obtention d'une paroi conductrice mobile. La paroi conductrice serait encore constituée par un cylindre d'aluminium A5 de faible épaisseur. En ce qui concerne la paroi mécanique, il faut noter que les matériaux à fort allongement n'ont jamais de résistance mécanique élevée; ils sont de plus très onéreux $(n / \varpi \simeq 3$ à 4 fois la valeur de l'aluminium A5 pour un acier inoxydable à forte résilience). Pour éviter cet inconvénient, on s'orienterait alors vers des aciers au carbone ordinaires ayant une bonne résistance mécanique et une faible résilience, en les rompant de manière contrôlée à l'arrivée de l'onde de choc. On aboutit ainsi au schéma de la figure 3 , où la cartouche en acier conte-
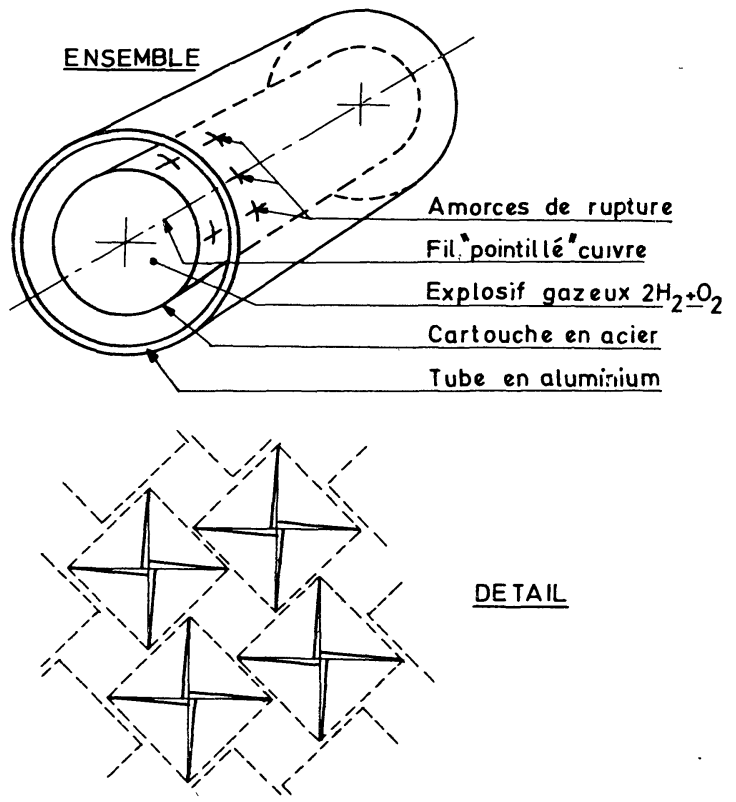

Fig. 3. - Exemple d'un système d'amorces de rupture facile à réaliser.

[An example of easy-to-realize grooves for initiating the rupture.]

nant le gaz s'ouvre sur toute sa surface pour laisser le passage aux gaz destinés à accélérer le liner conducteur. La structure proposée présente de multiples avantages :

- la cartouche en acier, faiblement accélérée, n'absorbe qu'un faible travail de déformation;

- le tube d'aluminium, qui ne supporte plus la pression, devient plus fin et sa vitesse plus grande;

- par analogie avec les observations faites avec les explosifs solides [4], le taux de dilatation du liner avant rupture sera plus grand, d'où la possibilité ultérieure d'accroître le rendement chimique ;

- le temps de délivrance de l'énergie sera plus court ;

- enfin, le prix de revient des parties détruites sera fortement réduit.

Au niveau 10 MJ, l'étude économique du générateur est un élément fondamental des choix constructifs. Aussi, nous adopterons dans la suite de l'étude ce perfectionnement.

Dans le projet qui suit, la vitesse du liner atteindrait $670 \mathrm{~m} / \mathrm{s}$, d'où une diminution très forte de la masse d'aluminium ( $15 \%$ de la masse d'un liner simple). La masse de la cartouche en acier serait du même ordre.

7.2 Relations ENTRE LES DIMENSIONS DU GÉNÉRATEUR. - Désignons par $(i)$ et $(f)$ les états initial et final du système. Pour que le rendement de conversion de l'énergie chimique soit le plus élevé possible, il faut permettre au tube de se dilater au maximum, c'est-à-dire jusqu'à l'apparition des fractures. 
Dans nos conditions expérimentales, cette limite est sensiblement atteinte [1,2] pour :

$$
\begin{aligned}
r_{0} & =25 e_{0} \\
r_{0 \mathrm{f}} & =1,6 r_{0 \mathrm{i}} .
\end{aligned}
$$

D'autre part, comme on recherche un fort coefficient de multiplication de l'énergie initiale, on aura :

$$
r_{\mathrm{of}} \simeq r_{2} .
$$

Cette dernière relation signifie que l'inductance initiale du générateur est d'environ $100 \mathrm{nH} / \mathrm{m}$.

Pour le rapport $r_{1 \mathrm{f}} / r_{1 \mathrm{i}}=1,6$, le rendement de détente de l'énergie chimique atteint $13 \%$ et l'énergie de déformation reste faible devant l'énergie cinétique. Dans ces conditions, une énergie finale de $10 \mathrm{MJ}$ sera obtenue à partir d'une énergie chimique initiale de $100 \mathrm{MJ}$ environ. Si nous adoptons une pression initiale du mélange de 60 bars (valeur courante expérimentalement), la densité d'énergie atteint $430 \mathrm{MJ} / \mathrm{m}^{3}$; le volume intérieur initial du liner serait alors de $0,25 \mathrm{~m}^{3}$.

Pour modérer les effets de longueur finie du liner. la longueur $h$ du liner doit satisfaire la relation

$$
h \gg r_{0}
$$

( $h \simeq 10 r_{0}$ en pratique)

Pour obtenir une énergie magnétique finale d'au moins $10 \mathrm{MJ}$, il faut alors satisfaire la relation :

$$
2 \pi r_{\mathrm{f}}\left(r_{2}-r_{\mathrm{f}}\right) h \cdot \frac{B_{\mathrm{f}}^{2}}{2 \mu_{0}} \geqslant 10^{7} .
$$

D'autre part, pour limiter la température de surface, nous conviendrons de borner $B_{\mathrm{f}}$ à 40 teslas. Alors, (25) devient :

$$
r_{\mathrm{f}}\left(r_{2}-r_{\mathrm{f}}\right) h \gtrsim 2,5 \times 10^{3} .
$$

Pour que les défauts de symétrie du liner, mis én évidence précédemment [2], n'affectent pas le fonctionnement du générateur, nous nous placerons dans des conditions initiales telles que la distance d'arrêt du liner à là carcasse soit de l'ordre de $5 \mathrm{~mm}$ :

$$
r_{2}-r_{\mathrm{f}} \simeq 5 \times 10^{-3} \text {. }
$$

7.3 Caractéristiques DU SyStÈMe PROPOSÉ. L'énergie chimique initiale vaut 107 MJ. Le liner a une masse de $54 \mathrm{~kg}$. Ses dimensions sont :

$$
\begin{array}{ll}
h=5 \mathrm{~m}, & r_{0}=0,125 \mathrm{~m}, \\
r_{1}=0,13 \mathrm{~m}, & r_{2}=0,21 \mathrm{~m} .
\end{array}
$$

La cartouche en acier, de $2 \mathrm{~mm}$ d'épaisseur, est placée à un rayon $r_{\mathrm{A}} \simeq 0,11 \mathrm{~m}$; ses dimensions précises n'influent d'ailleurs que faiblement sur les performances.

A la dilatation maximale, le jeu entre le liner et la paroi n'excède pas $5 \mathrm{~mm}$.
Le travail de détente de la vapeur, de $14 \mathrm{MJ}$, se décompose en $2 \mathrm{MJ}$ perdus dans le travail de déformation et $12 \mathrm{MJ}$ cinétiques (la vitesse atteint alors $670 \mathrm{~m} / \mathrm{s}$ ).

L'inductance initiale est de $480 \mathrm{nH}$. Elle est supérieure à $20 \mathrm{nH}$ en fin de compression.

8. Fonctionnement du générateur 10 MJ. - Nous avons calculé, à l'aide de la méthode de calcul numérique décrite précédemment, les performances du générateur dont nous venons de définir les dimensions.

Les figures 4 et 5 présentent les évolutions du rayon extérieur du liner $r(t)$, de la multiplication du courant $I / I_{0}$, de la multiplication de l'énergie magnétique $W / W_{0}$ et du coefficient de conservation du flux $\lambda=\Phi / \Phi_{0}$, à partir de deux valeurs différentes de l'énergie magnétique initiale.

Dans le premier cas considéré (correspondant à la figure 4)

- l'énergie initiale est : $W_{0}=1 \mathrm{MJ}$,

- le courant initial est : $I_{0}=2,05$ MA.

Nous avons représenté pour ce cas les variations temporelles du champ magnétique à la surface du liner $B(t)$ et la vitesse $V(t)$ du liner (Fig. 6).

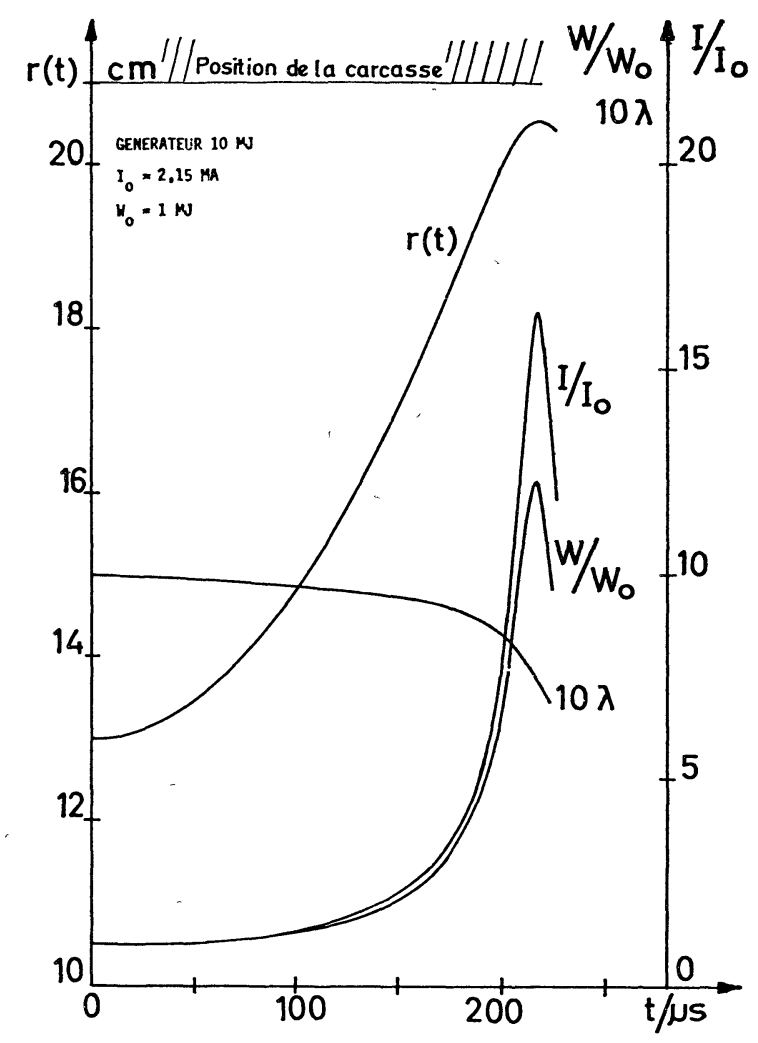

FIG. 4. - Evolution calculée du rayon $r(t)$ du liner, de l'accroissement relatif du courant $\left(I / I_{0}\right)$ et de l'énergie magnétique $\left(W / W_{0}\right)$, ainsi que du coefficient $\lambda$ de conservation du flux. Energie initiale : $1 \mathrm{MJ}$.

[Calculated evolution of the liner radius $r(t)$, of the current and magnetic energy relative increase $I / I_{0}$ and $W / W_{0}$, and of the flux conservation coefficient $\lambda$. The initial energy is $1 \mathrm{MJ}$.] 


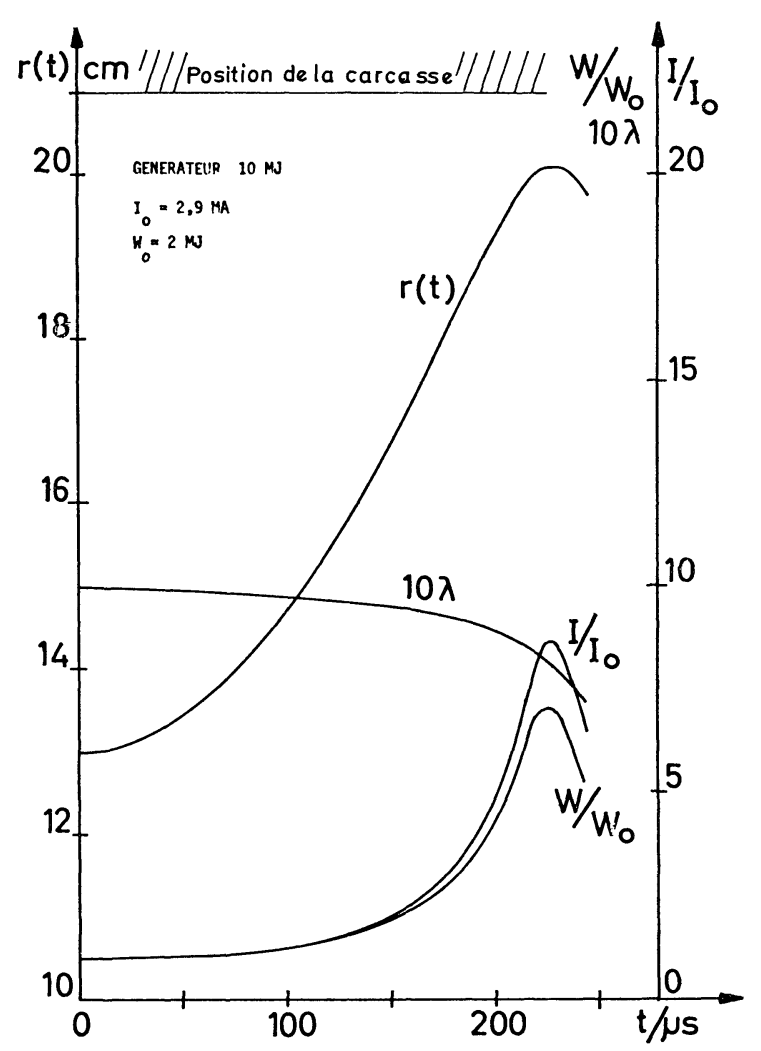

Fig. 5. - Evolution calculée du rayon $r(t)$ du liner, de l'accroissement relatif du courant $\left(I / I_{0}\right)$ et de l'énergie magnétique $\left(W / W_{0}\right)$, ainsi que du coefficient $\lambda$ de conservation du flux. Energie initiale $2 \mathrm{MJ}$.

[Calculated evolution of the liner radius $r(t)$, of the current and magnetic energy relative increase $I / I_{0}$ and $W / W_{0}$, and of the flux conservation coefficient $\lambda$. The initial energy is $2 \mathrm{MJ}$.]

Au début de la compression du champ magnétique (pendant les 150 premières microsecondes environ) le courant et l'énergie magnétique croissent lentement. Le champ magnétique reste pratiquement constant tandis que le liner subit une accélération presque constante (Fig. 6). Ensuite les croissances du courant et de l'énergie magnétique sont très rapides jusqu'à leur maximum.

$\mathrm{La}$ vitesse maximum du liner $\left(600 \mathrm{~m} . \mathrm{s}^{-1}\right)$ est atteinte pour $t=180 \mu$ s (l'énergie cinétique correspondante est 9,8 MJ); à cet instant l'énergie magnétique initiale a été multipliée par 3,6.

La croissance rapide du champ magnétique freine ensuite brutalement le liner qui s'arrête à $t=218 \mu \mathrm{s}$. Le champ magnétique, le courant et l'énergie magnétique sont maximum très peu avant l'arrêt du liner, ils atteignent respectivement 34,4 T, 34 MA, 12,3 MJ. Les pertes de flux par diffusion du champ magnétique sont de $25 \%$. La distance d'arrêt du liner à la paroi de la carcasse est de $5 \mathrm{~mm}$, l'inductance finale de $22 \mathrm{nH}$.

Au cours de ce fonctionnement la multiplication du courant et la multiplication de l'énergie magnétique atteignent respectivement 16,5 et 12,3 , tandis que la variation relative d'inductance est $L_{0} / L_{\mathrm{f}}=22$.

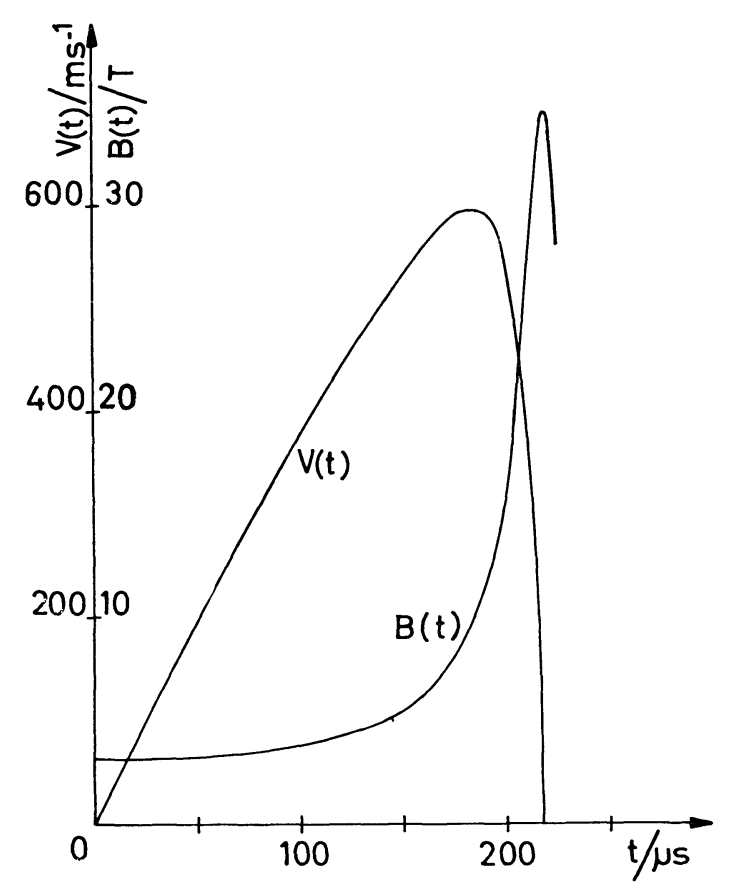

Fig. 6. - Evolutions calculées de la vitesse du liner et du champ magnétique à la surface, l'énergie magnétique initiale étant de $1 \mathrm{MJ}$.

[Calculated evolution of the liner velocity and of the superficial magnetic field. The initial energy is $1 \mathrm{MJ}$.]

Dans le deuxième cas de fonctionnement (Fig. 5)

- l'énergie magnétique initiale est $W_{0}=2 \mathrm{MJ}$,

- le courant correspondant est $I_{0}=2,9$ MA.

Les valeurs maximum du courant et de l'énergie magnétique ( $25 \mathrm{MA}, 14 \mathrm{MJ}$ ) sont atteintes à $t=227 \mu \mathrm{s}$. Les pertes de flux sont réduites : $18 \%$. Les multiplications du courant et de l'énergie magnétique, respectivement de 8,66 et de 7,1 , sont plus faibles que dans le premier cas; cependant la distance d'arrêt du liner à la carcasse est le double du cas précédent : $1 \mathrm{~cm}$. L'inductance finale est de $45 \mathrm{nH}$.

Le choix entre ces deux fonctionnements du générateur, qui aboutissent pratiquement à la même énergie finale, dépend à la fois des conditions d'emploi et de la distance d'arrêt minimum du liner à la carcasse que l'on peut accepter.

9. Influence de la charge. - Le générateur que nous venons de décrire est à très basse impédance; dans notre analyse du fonctionnement, le champ final est directement piégé à l'intérieur de l'ensemble linercarcasse. Au point de vue pratique, le fonctionnement sur une charge extérieure peut s'effectuer de 2 manières principales, représentées sur la figure 7 .

Lorsque la charge est une inductance fixe ou variable à faible impédance, il suffit de la connecter directement aux bornes du système. Pour réduire au maximum le flux résiduel entre la carcasse et le liner, ce dernier doit s'approcher très près. Le premier cas de fonctionnement $\left(W_{0}=1 \mathrm{MJ}\right)$ représente alors probable- 

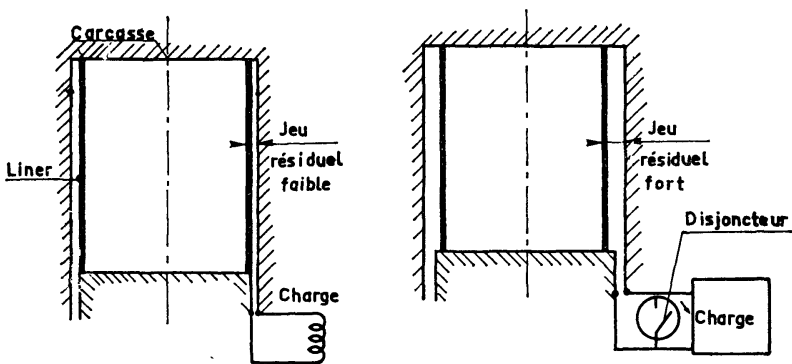

FIG. 7. - Position du liner en fin de compression pour deux utilisations caractéristiques.

[The liner position at the end of the compression for two characteristic uses.]

ment les performances limites de ce générateur, tandis que le deuxième cas $\left(W_{0}=2 \mathrm{MJ}\right)$ paraît plus raisonnable. En effet, lorsque l'on cherche des taux de compression élevés, les parties du liner en avance heurtent la paroi de la carcasse et une partie de l'énergie cinétique acquise par le liner est perdue; il en résulte une diminution' de l'énergie magnétique finale.

Une utilisation plus universelle valable pour toutes les charges, consiste à placer aux bornes du générateur un dispositif disjoncteur rapide [5] qui s'ouvre lorsque le liner est arrêté par la compression du flux magnétique interne invariant. Notons que les dispositifs à feuilles explosées sont souvent bien adaptés pour faire directement fonction de disjoncteur; ils permettent alors de réduire par un facteur de l'ordre de 5 le temps d'impulsion de l'énergie par rapport à une inductance fixe $[1,7]$. A priori, ce mode de fonctionnement présente d'importantes pertes au niveau du disjoncteur mais le liner reste toujours très éloigné de la carcasse : on peut se permettre alors un taux de compression correspondant au premier cas de fonctionnement $\left(W_{0}=1 \mathrm{MJ}\right)$.

Finalement, le choix du mode de couplage du générateur à la charge ne modifie pas notoirement le fonctionnement du générateur, mais intervient en ce qui concerne l'énergie initiale nécessaire.

10. Injection de l'énergie initiale. - Le fonctionnement du générateur à un niveau d'énergie finale de 10 MJ nécessite une source d'énergie de l'ordre de $1 \mathrm{MJ}$ dans l'inductance initiale $(480 \mathrm{nH})$ du générateur. Une batterie de condensateurs est a priori bien adaptée aux conditions requises et ne présente pas de difficultés de mise en œuvre puisque l'on ne recherche pas des temps de décharge très brefs. Cependant, les avantages que présente le générateur à explosif gazeux ne peuvent pas être entièrement exploités avec un banc de condensateurs de $1 \mathrm{MJ}$. En effet, il alourdit considérablement le dispositif et il demande un investissement de l'ordre de 1 million de francs, c'est-à-dire bien supérieur au coût du générateur lui-même. Aussi, il est indispensable de trouver un procédé de fourniture de l'énergie initiale moins onéreux.
Une solution peu coûteuse a priori consiste à créer le champ primaire par une génératrice homopolaire sans fer [8]. Mais la structure à courant axial retenue ne se prête pas à une telle solution car la résistance électrique du liner est trop élevée : il faudrait alors remplacer la carcasse lisse par une bobine.

Une autre solution, que nous retenons, consiste à utiliser des générateurs à explosif montés en cascade, ce qui a pour effet tout à la fois de réduire les efforts sur les structures et surtout d'accroître le taux de compression apparent. Avec seulement deux générateurs en cascade, on peut réduire par un facteur de l'ordre de 5 le prix de la batterie de condensateurs nécessaire, ce qui résout le problème du coût de la source primaire. Notons toutefois qu'un problème d'adaptation d'impédance se pose alors : l'inductance finale du premier générateur doit être l'inductance initiale du second. Pour le résoudre, on dispose de deux méthodes principales. La première consiste à fractionner le générateur aval en plusieurs générateurs élémentaires (5 à 10) montés en parallèle (Fig. 8).

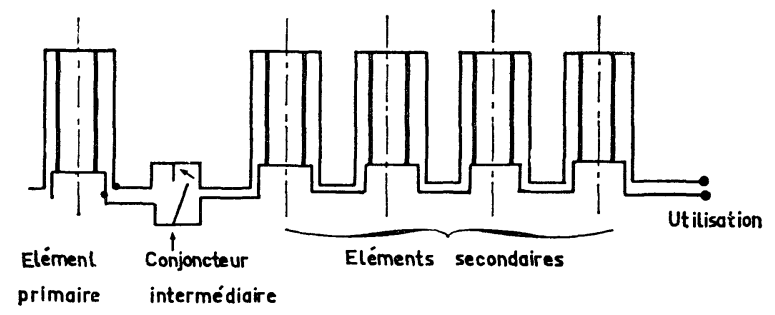

FIG. 8. - Structure d'un générateur en cascade sans adaptation d'impédance.

[The structure of a multi-generator without impedance adaptation.]

La seconde consiste à placer entre les deux générateurs un transformateur d'impulsion (Fig. 9). Si le transformateur est classique, c'est-à-dire à noyau de fer, l'énergie transférée peut atteindre quelques $\mathrm{kJ} / \mathrm{dm}^{3}$ de fer en effectuant une polarisation initiale inversée de l'aimantation; le rendement est très élevé (supérieur à $90 \%$ ) [9]. Au prix d'un rendement plus faible mais avec un investissement inférieur, il est également possible d'employer un transformateur sans fer. Dans ce cas, l'énergie transférable atteint $10 \mathrm{~kJ} / \mathrm{dm}^{3}$ de cuivre [10].

Finalement, en tenant compte à la fois des investissements et de la facilité de mise en œuvre, nous nous sommes orientés vers un générateur constitué de 2 liners en cascades connectés par un transformateur

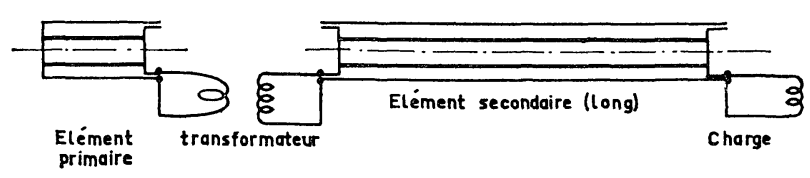

FIG. 9. - Structure d'un générateur à adaptateur d'impédance.

[The structure of an adaptated impedance generator.] 
sans fer. C'est à partir de cette solution que nous évaluóns le prix auquel reviend rait une telle réalisation.

11. Evaluation du coût du générateur. - Examinons succinctement les aspects économiques de la construction et de l'exploitation du générateur à explosif gazeux de $10 \mathrm{MJ}$ d'énergie finale que nous venons de décrire. Comparons-les aux coûts des bancs de condensateurs et des générateurs à explosif solide de même niveau d'énergie.

La simplicité technologique du générateur à explosif gazeux permet une construction bon marché. On peut estimer son coût d'après sa masse totale, celle-ci étant essentiellement constituée par la carcasse en acier. Le générateur, délivrant $10 \mathrm{MJ}$, nécessitant ùne carcasse de l'ordre de 5 tonnes, on peut évaluer le coût de la réalisation à environ $200000 \mathrm{~F}$.

En fait, la plus grande part de l'investissement est demandée par la source primaire d'énergie. Si l'on considère le montage en cascade, la source primaire d'énergie comprend :

- une batterie de condensateurs de $200 \mathrm{~kJ}$ : $400000 \mathrm{~F}$,

- le premier générateur coaxial délivrant une énergie de $1 \mathrm{MJ}$ et dont la masse est de l'ordre de 1 tonne : $30000 \mathrm{~F}$,

- le transformateur d'impulsion dont la masse des enroulements en cuivre est voisin de 1 tonne : $400000 \mathrm{~F}$.

On évalue ainsi grossièrement l'investišsement global nécessaire à la réalisation de cet ensemble à $1000000 \mathrm{~F}$ environ.

On peut comparer ce chiffre à l'investissement demandé pour un générateur à explosif solide de même énergie qui s'élève aujourd'hui à $3000000 \mathrm{~F}$ environ. Le coût d'un banc de condensateurs équivalent est bien supérieur encore, de l'ordre de 20 millions de francs.
Sur le plan économique, l'utilisation d'un générateur à explosif gazeux réduit donc considérablement les investissements; gain d'un facteur 3 sur les dispositifs à explosif solide, d'un facteur 20 sur les bancs de condensateurs.

Le coût de fonctionnement du générateur à explosif gazeux provient pour l'essentiel du prix des parties détruites à chaque tir ; le coût de l'explosif (mélange $2 \mathrm{H}_{2}+\mathrm{O}_{2}$ obtenu par électrolyse d'une solution de potasse) est négligeable, inférieur à $1 \mathrm{~F} / \mathrm{MJ}$ utile. Le prix du liner en aluminium et de la cartouche en acier contenant l'explosif gazeux sous pression est faible. L'aluminium est fourni à $20 \mathrm{~F} / \mathrm{kg}$ et est repris à $13 \mathrm{~F} / \mathrm{kg}$, le prix de la cartouche en acier est d'environ $10 \mathrm{~F} / \mathrm{kg}$; ainsi à chaque tir $1000 \mathrm{~F}$ environ de matériaux sont perdus.

Comparons ce coût d'exploitation à celui des générateurs à explosif solide : $20000 \mathrm{~F}$ (en 1969) pour chaque tir d'après $H$. Knoepfel [11]; l'explosif gazeux permet d'abaisser considérablement cette valeur, d'un facteur 30 .

12. Conclusion. - Nous venons d'effectuer la conception d'un générateur impulsionnel de grande énergie (10 MJ) capable de délivrer un courant de l'ordre de $30 \mathrm{MA}$ en $100 \mu$ s environ. Un des principaux domaines d'application de ce générateur serait l'alimentation d'expériences fusion thermonucléaire contrôlée à confinement inertiel. L'investissement à envisager serait de l'ordre de $10^{6} \mathrm{~F}$, soit 3 fois moins qu'un générateur à explosif solide (détruit à chaque tir) et 20 fois moins qu'un banc de condensateurs équivalent. Son coût de fonctionnement, $10^{3} \mathrm{~F}$ par tir, reste très faible. Ces générateurs présenteraient donc, sur le plan économique, un grand intérêt dans toutes les applications nécessitant la fourniture brutale d'une grande énergie impulsionnelle.

\section{Bibliographie}

[1] Knoepfel, H., Kroegler, H., Luppi, R., VAn Monfort, J., Rev. Sci. Instrum. 40 (1969) 60.

[2] Hahn, R., Antoni, B., Lucidarme, J., Rioux, C., RiouxDamidau, F., Rev. Phys. Appl. 11 (1976) 409.

[3] Richtmyer, R. D., Morton, K. W., Difference Methods for Initial Value Problems (Interscience Publishers), 1967.

[4] Slate, P. M. B., Billings, M. J. W., Fuller, P. J. A., J. Inst. Met. 95 (1967) 244.

[5] Somon, J. P., Thèse de Doctorat Université Paris XI (1968)

[6] Rioux, C., Rioux-Damidau, F., Rev. Phys. Appl. 9 (1974) 539.
[7] Antoni, B., Thèse de Doctorat Université Paris XI (1974).

[8] Guillet, R., Hahn, R., Lucidarme, J., Rioux, C., Rev. Phys. Appl. 8 (1973) 53.

[9] Rioux, C., Rapport interne LF 46 (1975).

[10] Furth, H. P., Waniek, R. W., Proceeding of the Conference on Megagauss Magnetic Field Frascati (1965).

[11] Knoepfel, H., Kroegler, H., Luppi, R., Verbeek, R., VI Symp. Fusion Technologie AACHEN (septembre 1970).

[12] Boissady, C., Postel, C. and Rioux-Damidau, F., J. Appl. Phys. 48 (1977) 1880 